\title{
Transformer les vagues en énergie : utopie ou réalité ?
}

Turning waves into energy: a utopian dream or a reality?

Sylvain Roche, Alain H. Clément, Aurélien Babarit et Christophe Bouneau

\section{(2) OpenEdition}

Journals

Édition électronique

URL : http://journals.openedition.org/artefact/3352

DOI : $10.4000 /$ artefact.3352

ISSN : 2606-9245

Éditeur :

Association Artefact. Techniques histoire et sciences humaines, Presses universitaires du Midi

Édition imprimée

Date de publication : 15 mars 2019

Pagination : 239-265

ISBN : 978-2-8107-0623-5

ISSN : 2273-0753

Référence électronique

Sylvain Roche, Alain H. Clément, Aurélien Babarit et Christophe Bouneau, «Transformer les vagues en énergie : utopie ou réalité ? ", Artefact [En ligne], 9 | 2018, mis en ligne le 04 mars 2020, consulté le

10 décembre 2020. URL : http://journals.openedition.org/artefact/3352 ; DOI : https://doi.org/

10.4000/artefact.3352

Artefact, Techniques, histoire et sciences humaines est mise à disposition selon les termes de la Licence Creative Commons Attribution - Pas d'Utilisation Commerciale - Pas de Modification 4.0 International. 


\section{Transformer les vagues en énergie : utopie ou réalité ?}

\section{Sylvain Roche, Alain H. Clément, Aurélien Babarit et Christophe Bouneau}

\section{Résumé}

Cet article s'intéresse à la trajectoire contrariée de l'énergie des vagues. S'inscrivant dans une démarche forecasting volontairement transdisciplinaire à la croisée des sciences sociales et des sciences de l'ingénieur, notre propos vise à questionner la situation présente (insight) de cette énergie en replaçant les enjeux à travers le prisme rétrospectif (hindsight) et prospectif (foresight). Comment expliquer la difficulté du houlomoteur à se déployer industriellement alors que la recherche scientifique est foisonnante depuis le $x_{1 \times}{ }^{e}$ siècle ?

\section{Mots-clés}

énergie des vagues, énergies marines renouvelables, innovations de rupture, promesses technologiques, processus d'innovation, verrous technologiques, utopie technologique

99 Sylvain Roche, Alain H. Clément, Aurélien Babarit et Christophe Bouneau, «Transformer les vagues en énergie : utopie ou réalité ? », Artefact, 9, 2018, p. 239-265. 


\section{Turning waves into energy: a utopian dream or a reality?}

\section{Abstract}

This paper will examine the complicated path of wave energy with a transdisciplinary and forecasting approach between social and engineering sciences. Our statement is to question the present situation of wave energy regarding hindsight and foresight aspects. How to explain wave energy's difficulties for industrial implementation while scientific research is abundant since the $19^{\text {th }}$ century?

\section{Keywords}

wave energy, marine renewable energies, breakthrough innovations, technological promises, innovation process, technology locks, technological utopianism 


\section{Introduction}

La fascination pour les vagues inspire les artistes depuis le $\mathrm{XIX}^{\mathrm{e}}$ siècle les artistes, les conduisant souvent à des conduites extrêmes ${ }^{1}$. Elles ont depuis longtemps frappé les imaginations, provoquant un sentiment de liberté et de puissance, faisant éprouver à l'homme sa finitude et sa faiblesse. Comment ici ne pas penser aux vagues de Gustave Courbet ou à celles de Hokusai ? Ou encore aux vagues géantes, les vagues scélérates, qui alimentent depuis des siècles l'imaginaire des marins ? Cette image de la force des vagues dans l'inconscient collectif se nourrit aussi des résultats des catastrophes naturelles de type tsunami.

À côté de cet imaginaire maritime se développe un imaginaire autour de l'énergie. Depuis la révolution industrielle et l'avènement de la fée électricité, la production et les transformations de l'énergie sont un terrain de chasse privilégié pour la construction des imaginaires collectifs ${ }^{2}$. Le $\mathrm{XX}^{\mathrm{e}}$ siècle a vu ainsi s'enchaîner toute une palette de technologies présentées bien souvent comme "la " solution définitive aux diverses crises énergétiques, mettant en exergue la fragilité de notre système énergétique ${ }^{3}$. On connaissait l'empire britannique et son hymne Britannia rule the waves, qui rêvait de commander aux vagues. Grâce à la technologie, les ingénieurs entendent bien réaliser ce rêve en produisant de l'énergie à foison ${ }^{4}$. Par son fort pouvoir cognitif et communicationnel (l'énergie de la houle est une puissance mentalement simple à se représenter), la vague symbolise naturellement les "énergies nouvelles ", et plus spécifiquement la filière des énergies marines dont elle devient l'emblème (Fig. V, cahier couleur).

\footnotetext{
1. À ce titre, le Musée d'art moderne André Malraux (MuMa) du Havre a présenté en 2004 deux expositions «Vagues 1. Autour des Paysages de mer » et "Vagues 2. Hommages et digressions » afin de montrer les divers aspects, poétiques, artistes, fantasmagoriques, propres à la vague. Voir Annette Haudiquet, Marc Donnadieu, Jean-Pierre Melot, Vagues II - Hommages et digressions, catalogue d'exposition - Le Havre, Musée d'art moderne André Malraux, 26 juin-27 septembre 2004, Paris, Somogy, 2004.

2. Alain Beltran, Patrice Carré, La Vie électrique, Histoire et imaginaire (XVIT'-XXI siècle), Paris, Belin, 2016.

3. Jean-Claude Debeir, Jean-Paul Deleage, Daniel Hemery, Une Histoire de l'énergie, Paris, Flammarion, 2013.

4. "L'île qui veut commander aux vagues ", Presse-Océan, 24 avril 2008.

5. Un long plan d'une énorme vague est diffusé dans le journal d'Antenne 2 le Midi ( $1^{\text {er }}$ août 1979) pour présenter les recherches françaises dans le domaine des énergies nouvelles.
} 
Dans cette histoire où la mer se présente comme la nouvelle frontière à conquérir, l'énergie des vagues participe à la construction d'un imaginaire $\mathrm{du}$ fait de son insertion dans cet univers symbolique : elle matérialise un nouveau projet technologique, cette mer $\mathrm{du} \mathrm{XxI}$ siècle source d'énergies renouvelables inépuisables ${ }^{6}$, ce grand rêve pour demain dont témoignent de nombreux articles : "Il y a longtemps que l'on rêve d'exploiter l'énorme potentiel d'énergie que recèlent les vagues ${ }^{7}$ "; "Les vagues ne font pas rêver que les surfeurs. Depuis plus de vingt ans, les chercheurs veulent utiliser cette source considérable d'énergie pour produire de l'électricité ${ }^{8}$ ". Le prisme énergétique fait ainsi passer les vagues du symbole du chaos à celui de l'abondance?.

Comme pour toute technologie émergente, l'énergie des vagues suscite néanmoins toute une série d'hypothèses et de spéculations sur les impacts économiques et industriels qu'elle peut générer, et ceci depuis les années 1970, sans compter les nombreux verrous technologiques encore non résolus. Cet article se propose ainsi de mieux comprendre le développement de la filière houlomotrice et ses difficultés actuelles à passer d'une phase académique à une phase industrielle. Une mise en perspective historique nous permettra d'examiner le processus d'innovation et de maturité technologique en prenant l'énergie des vagues comme cas d'étude. Pourquoi la filière houlomotrice est-elle restée à l'état de curiosité de laboratoire pour certains et de réalité concrète pour d'autres?

Notre analyse se fera volontairement sur un temps long afin de dégager les grandes dynamiques et les phases séquentielles de l'énergie des vagues. Centré sur le cas français, notre propos gardera constamment un regard sur les dynamiques étrangères pour interroger la diversité des expériences locales et nationales. Pour finir, nous insistons sur le fait que cet article n'est pas une tribune pour défendre la filière houlomotrice et ses acteurs promotionnels. Il souligne uniquement une volonté commune de questionner

6. Cet engouement pour cette filière peut aussi s'expliquer par la « qualité de la ressource ». Si elles pouvaient être converties en électricité, les vagues qui déferlent en continu sur la façade atlantique de la France fourniraient 420 térawattheures par an, soit l'équivalent de $90 \%$ de la consommation électrique annuelle. http://wikhydro.developpement-durable.gouv.fr/index.php/Energies_marines, consulté le 7 novembre 2018.

7. «Énergies des vagues, une palette de solutions en cours d'exploitation ou d'essai ", Marine, janvier 2006, $\mathrm{n}^{\circ} 210$.

8. "Ces vagues qui font rêver les chercheurs ", TerraEco, 2 juillet 2009.

9. «La houle aux œufs d'or », Les Report Terre, France 5, 2 juillet 2009. 
la situation présente (insight) de cette filière en replaçant le débat actuel à travers le prisme rétrospectif (hindsight) et prospectif (foresight). Cette intégration d'une double perspective " rétrospective/prospective ", qui s'inscrit parfaitement dans le domaine pluridisciplinaire des sciences, technologies et société (STS), pourra ainsi conduire à des conclusions différentes, des regards et des interprétations divers concernant l'avenir de cette filière située aujourd'hui à la croisée des chemins.

\section{Le temps de la créativité et du machinisme triomphant (1799-1974)}

"Capter cette formidable énergie gratuite des vagues ${ }^{10}$ " est " un rêve tenace de l'humanité ${ }^{11}$ ». De nombreux ingénieurs et chercheurs ont eu de tout temps les yeux rivés sur ce que les marins appellent « la mer du vent». La révolution technique anglaise du XviII ${ }^{\mathrm{e}}$ siècle et l'essor du machinisme sont un facteur essentiel dans cette histoire car ils ont bouleversé la façon de voir le monde et l'élément marin. Le machinisme redéfinit les images classiques que l'on avait de la mer, façonnées au cours des siècles. La mer devient une opportunité pour l'homme technicien, et à ce titre, la vague apparaît non plus comme un danger mais comme une ressource naturelle à maîtriser. C'est dans ce contexte où la machine à vapeur est reine que des " expérimentateurs éclairés ${ }^{12}$ " proposent l'idée d'exploiter l'énergie des vagues à la fin du XIX ${ }^{\mathrm{e}}$ siècle. Le plus ancien brevet connu visant à l'utilisation de l'énergie des vagues est déposé à Paris par les Girard, père et fils, le 12 juillet $1799^{13}$.

La fin du XIX ${ }^{e}$ siècle est marquée par les applications sans cesse grandissantes autour de l'électricité. L'électricité introduit de nouvelles machines et dessine un nouvel imaginaire technologique. Le monde de la mer n'échappe pas à la fée électricité. Le titre du chapitre XII de Vingt mille lienes sous les mers (1870) de Jules Verne sonne ici comme un manifeste : "Tout par l'électricité ». À la même époque, Victor Hugo écrit dans son roman Quatrevingt-treize (1874) : «Réfléchissez au mouvement des vagues, au flux

10. Dans « Les énergies des mers ", Clés pour demain, 19 janvier 1981.

11. Sciences et Avenir, hors-série "La conquête de la planète Océan ", mai 1986.

12. Jean-Pierre Rioux, La Révolution industrielle, 1780-1880, Seuil, Paris, 1971, p. 61.

13. David Ross, Power from the Waves, Oxford, Oxford University Press, 1996, p. 8. 
et reflux, au va-et-vient des marées. Qu'est-ce que l'océan ? Une énorme force perdue. Comme la terre est bête ! Ne pas employer l'océan! ». Dans cette fin $\mathrm{du} \mathrm{XIX}^{\mathrm{e}}$ siècle où la mer devient progressivement un « laboratoire fertile pour l'expérimentation ", la vague apparaît comme un défi ${ }^{14}$.

Devant répondre aux nouvelles exigences sécuritaires, les littoraux mondiaux se maillent de phares et de balises. Des artefacts houlomoteurs sont imaginés pour rendre les sémaphores autonomes. Ces derniers s'inspiraient des bouées Courtenay, inventées aux États-Unis, qui par le mouvement vertical de la vague permettaient de comprimer l'air qui passait par un sifflet pour obtenir une bouée sonore ${ }^{15}$. La fin du XIx ${ }^{\mathrm{e}}$ siècle est en effet une période qui voit la multiplication des expériences technologiques avec l'air comprimé ${ }^{16}$. En France, le Service des phares et balises trouve la parade en installant sur les bouées une cloche ou un sifflet, actionnés par la houle, pour signaler les dangers isolés.

Cette période de profusion d'innovations où les applications de l'électricité ne semblent connaître aucune limite encourage des amateurs et bricoleurs ingénieux à se lancer dans d'ambitieux projets houlomoteurs financés sur des fonds propres. Tout comme les débuts de l'automobile, de l'aviation ou de l'informatique, l'énergie des vagues est au départ l'œuvre de pionniers tous aussi passionnés qu'originaux. On peut citer ici le projet de Wave motor de Victor Gauchez de 1882 ou encore celui de Franck Perry de 1906. En 1895, en Californie, Terrence Duffy créait la société WavePower Air-Compressing pour commercialiser un moteur à vagues ${ }^{17}$, lançant un engouement des Californiens pour les houlomoteurs qui perdura durant près de deux décennies. (Fig. 1) Selon David Ross, Thomas Edison lui-même aurait proposé une solution pour produire de l'électricité à partir des vagues à l'aide de dynamos ${ }^{18}$.

14. Annette Haudiquet, Marc Donnadieu, Jean-Pierre Melot, Vagues II - Hommages et digressions, op. cit.

15. Émile Allard, "Bouées sonores et bouées lumineuses. Note. № 27 ", 1882, Bibliothèque des Phares, http://bibliothequedesphares.fr/annales_ponts/annales_ponts_1882_0025, consulté le 23 janvier 2018.

16. Cyrille Simmonet, Brève histoire de l'air, Versailles, Quae, 2014.

17. Terrence Duffy, Duffy's wave motor as a source of power for various purposes, San Francisco, Francis, Valentine \& Co, 1886.

18. David Ross, Power from the Waves, op. cit., p. 9. 


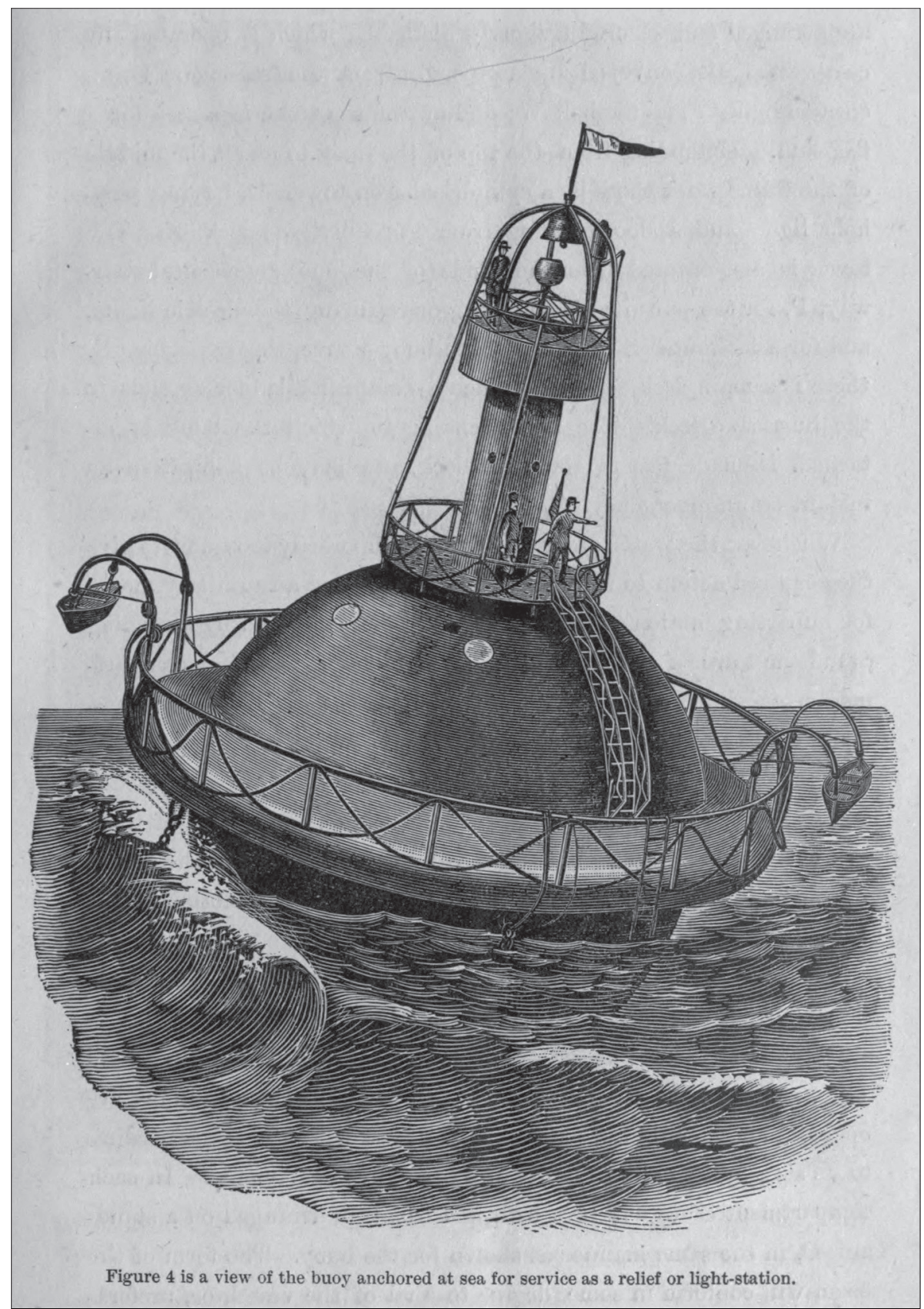

Fig. 1 - Bouée fonctionnant à l'énergie des vagues imaginée par Terence Duffy

Terrence Duffy, Duffy's wave motor as a source of power for various purposes, San Francisco, Francis, Valentine \& Co, 1886. 
Dans la France de l'entre-deux-guerres, l'énergie des vagues est marginalisée par rapport à l'énergie marémotrice, malgré les nombreuses demandes envoyées à la Commission de la houille bleue ${ }^{19}$ à ce sujet ${ }^{20}$. Des projets avant-gardistes aboutissent néanmoins et marquent de leur empreinte le littoral, là où les côtes rocheuses et abruptes présentent des cavités naturelles dans lesquelles les vagues s'engouffrent et compriment l'air. En 1910, près de Royan, Bouchaux-Pracéique eut l'idée d'aménager un de ces « trous du souffleur " pour installer une turbine à air attelée à une dynamo qui lui permettait d'éclairer sa maison. C'est ce principe que reprit, entre 1926 et 1929, André Coyne, un ingénieur du service des phares et balises. Ses articles dans la Revue générale de l'Hydraulique vont permettre de mieux faire connaître les potentialités de l'énergie de la houle. Il construisit à côté du phare du Minou, près de Brest, une sorte d'entonnoir artificiel dans lequel les vagues par effet de bélier comprimaient l'air ${ }^{21}$. Il songeait à une utilisation de cet air comprimé lorsque l'installation fut détruite par une tempête ${ }^{22}$. À la même époque, au pied du cap Saint-Martin à Biarritz, l'ingénieur Paul Grasset installait un prototype de "laboratoire hydrodynamique marin " afin de convertir l'énergie produite par la houle en électricitée 23 .

Câble tracteur. Soufflet compresseur. Marteau pneumatique. Pompes foulantes motrices. Roue dentée. Pompe foulante pneumatique. Tube à mercure... L'imagination des chercheurs ne fait pas défaut et de nombreux principes de captation de l'énergie des vagues sont proposés. Des réflexions sont même menées pour rendre (en partie) autonomes les navires, «la mer devenant motrice et poussant elle-même les bateaux qu'elle porte ${ }^{24}$ ».

Le $1^{\text {er }}$ janvier 1949 voit la mise en place d'un code international d'observation de la houle. Cette uniformisation encouragea les collaborations

19. La Commission de la houille bleue fut créée en 1919 pour se saisir de projets relatifs à l'utilisation de l'énergie des marées.

20. Anaël Marrec, «Politiques des forces naturelles et imaginaires de l'énergie pendant l'entredeux-guerres", Pour mémoire, 2016, n 18, p. 83-91.

21. André Coyne, "La captation de la houle ", Revue générale de l'Hydraulique, 3 avril 1939, p. 141-148.

22. Il donna néanmoins deux ou trois chevaux-vapeur.

23. Pierre Devaux, «Force motrice des vagues et de la houle. La nouvelle station d'essais d'hydrodynamique maritime du phare de Biarritz ", La Nature, 1934, $2^{\mathrm{e}}$ semestre, p. 260-266.

24. Maxime VINCENT, Réflexions sur l'utilisation future des énergies naturelles, vagues, chutes, hydrauliques et barométriques, Chaleur solaire, Paris, Librairie Fischbacher, 1924, p. 35. 
internationales dans le domaine de l'énergie des vagues et une meilleure diffusion des expérimentations à l'échelle mondiale. Dans cette période d'après-guerre, au Japon, le commandant Yoshio Masuda eut l'idée de remplacer le sifflet par un système de clapets et de turbine à air, couplée à une génératrice. Ce dispositif servit énormément pour le balisage des côtes japonaises. Dès lors, les Japonais se spécialisent sur la technique OWC (technique dite de colonne d'eau oscillante) et développent d'importants travaux de recherche menés à l'institut de recherche sur les ports (PHRI) par le professeur Takahashi, présenté à l'époque comme le père de l'houlomoteur dans le monde. En Angleterre, "Sir Alexandre Gibb and Partners » étudie un projet houlomoteur en 1959, à la suite d'une proposition du "Central Electricity Board ». C'est le projet " Île Maurice », dont la faisabilité technique s'est trouvée confirmée, mais non sa rentabilité25.

En France, des recherches sur la mesure des vagues et des dièdres à houle sont réalisées au laboratoire dauphinois d'hydraulique entre 1944 et $1948^{26}$. Ces études sont poursuivies jusque vers 1956 . Elles aboutissent à un avant-projet de centrale houlomotrice pour le site de Casablanca, sur la côte marocaine ${ }^{27}$. Le musée Océanographique de Monaco, sous l'impulsion du Dr Richard, expérimenta lui aussi un certain nombre de dispositifs destinés à capter l'énergie des vagues ${ }^{28}$. En juin 1956 se tiennent à Paris les IV Journées de l'hydraulique, organisées par la Société hydrotechnique de France et consacrées aux "Énergies de la Mer». Les exposés portaient presque exclusivement sur la houle, les vagues et les marées, témoignant déjà d'un intérêt certain du monde académique pour cette énergie ${ }^{29}$. En 1956, on dénombre plus de 600 brevets sur la question de l'houlomoteur représentant « 450 dispositifs à flotteurs assortis de dispositifs à panneaux, à pistons liquides et à plans inclinés ${ }^{30}$ ». Marquée par l'âge d'or

\footnotetext{
25. Pierre Guevel, "La récupération de l'énergie des vagues ", in Sciences et techniques de l'armement, Paris, Mémorial de l'artillerie française, 1986, t. 60, p. 21.

26. Maurice Gariel, «Énergie mécanique de la houle : utilisation ", La Houille Blanche, 1957, $\mathrm{n}^{\circ} 5$, p. 781.

27. Yves MaHÉ, "La Houle sur la côte atlantique du Maroc, in Les énergies de la mer. Compte des quatrièmes journées de l'hydraulique, Paris 13, 14, et 15 juin 1956, La Houille Blanche, 1957, p. 71-78.

28. Vsevolod Romanovsky, La Mer source d'énergie, Paris, PUF, 1950, p. 109.

29. Société hydrotechnique de France, Les Énergies de la mer. Compte rendu des quatrièmes Journées de l'hydraulique, Paris, 13, 14 et 15 juin 1956, La Houille Blanche, 1957.

30. Maurice Gariel, "Énergie mécanique de la houle : utilisation », op. cit., p. 780.
} 
des barrages hydroélectriques et des études hydrodynamiques sur les courants de marées, la France fait le choix de concentrer ses efforts sur le développement de l'énergie marémotrice, mieux maîtrisée, plus productive et répondant grâce à sa prédictibilité à la logique centralisée du modèle EDF, aboutissant à l'inauguration de l'usine de la Rance en novembre 1966. «Il y a peu à espérer, semble-t-il, de l'utilisation des vagues et de la houle. Toutes les inventions proposées jusqu'ici, tous les essais entrepris montrent qu'on n'en peut attendre que de faibles puissances, très inconstantes, aux prix de très coûteuses installations. Plus tentante est l'utilisation des marées ${ }^{31}$ ". Cette dynamique portée par les projets marémoteurs (Rance et Chausey) encourage néanmoins les études autour de l'énergie de vagues : "La houle joue un rôle considérable dans la réalisation d'un projet comme Chausey et nos connaissances sur ce sujet sont encore bien imparfaites ${ }^{32}$ ".

Jusqu'au début des années 1970, les dispositifs construits se sont essentiellement soldés par des échecs, les frêles constructions ne pouvant avoir raison des tempêtes ${ }^{33}$. C'est "l'âge de la préhistoire ${ }^{34}$ " pour l'énergie des vagues, se résumant à une histoire de la science de la mesure des vagues (climatologie de la houle) cantonnée à rester un doux rêve d'ingénieur : "L'énergie des vagues ne peut, en aucun cas, se comparer ni à l'énergie thermique des mers, ni à celle que l'on peut espérer retirer des variations dues à la marée. Elle restera dans le domaine des curiosités sans application industrielle ${ }^{35}$ ". Remarquons néanmoins que dès la fin des années 1950, tous les grands principes de récupération de l'énergie des vagues sont déjà identifiés ${ }^{36}$.

31. René Legendre, «Les Ressources énergétiques de la Mer », Bulletin de l'Institut Océanographique, mars $1949, n^{\circ} 947$, p. 7.

32. Robert Gibrat, L'Énergie des Marées, Paris, PUF, 1966, p. 208.

33. Notons néanmoins la réussite du Wave motor américain qui a parfaitement fonctionné.

34. Aurélien Babarit, Ocean Wave Energy Conversion: Resource, Technologies and Performance, Londres, ISTE Press, 2017.

35. Vsevolod Romanovsky, La Mer source d'énergie, op. cit., p. 121.

36. Aurélien Babarit, Ocean Wave Energy Conversion, op. cit. 


\section{Le temps de l'invention et de la crise énergétique (1974-1998)}

Après le premier choc pétrolier de 1973, les financements publics de la recherche et développent consacrés à des projets de rupture des technologies de l'énergie sont perçus comme la meilleure façon de répondre aux externalités liées au pouvoir de marché de l'Opep. Cette crise encourage à impulser une dynamique vertueuse autour des " énergies nouvelles " et a pour effet de valoriser le prix « kWh-houle » par rapport au prix du « kWhdiesel ». L'évolution est palpable :

Dès les années 1940, les études entreprises en France sur le principe des dièdres conclurent à des coûts prohibitifs [...]. Cette étude reprise après les évènements de 1973-1974 est de ce fait très intéressante puisque la conclusion fait apparâtre que le prix du « kWh houle » est inférieur au prix du kWh diesel ${ }^{37}$.

Plusieurs pays comme la Norvège, le Japon, les États-Unis ainsi que le Royaume-Uni, mettent alors en ouvre des programmes de "technology push " d'ampleur variable, pour développer rapidement des technologies houlomotrices. L'objectif est double : dans un premier temps, préciser les potentiels houlomoteurs nationaux, puis dans un second temps développer des systèmes d'exploitation aptes à la commercialisation. Ces programmes ont en commun la volonté de s'appuyer sur les retours d'expérience issus de l'industrie pétrolière offshore et de l'industrie navale ${ }^{38}$.

L'apport de ces nouveaux savoirs industriels permet de plus au houlomoteur de se "scientifiser". Un certain nombre de chercheurs académiques examinent l'énergie des vagues dans cette période, parmi lesquels Stephen Salter de l'Université d'Edimbourg ${ }^{39}$, Kjell Budal et Johannes Falnes de l'Institut norvégien de technologie, ou encore David Evans de l'Université de Bristol. En France, l'étude de la récupération de l'énergie des vagues s'émancipe du marémoteur et devient un sujet de travail universitaire en

37. Gilbert Damy, Michel Gauthier, Production d'énergie à partir de la houle, Ifremer, CNEXOCOB, 1981, p. 35.

38. Gilles Susbielles, Christian Bratu, Alain Cavanie, Vagues et ouvrages pétroliers en mer, Paris, Technip, 1981.

39. L'article «Waver power » de Stephen Salter dans la revue Nature en 1974 fait entrer définitivement l'énergie des vagues dans la sphère scientifique. 
entrant dans les laboratoires de l'École nationale supérieure de mécanique (ENSM) de Nantes, ancêtre de l'actuelle École centrale de Nantes. Ce processus de scientifisation entraîne l'apparition d'une communauté scientifique dédiée au houlomoteur à l'échelle internationale, permettant le partage des connaissances et le développement de l'intelligence collective. En 1979 le premier colloque scientifique international sur l'énergie des vagues a lieu à Göteborg (First International Symposium on Wave Energy Utilization). Il met en réseau la communauté scientifique. L'ENSM de Nantes noue alors des relations à l'international, et notamment avec les chercheurs de l'Instituto Superior Tecnico de Lisbonne.

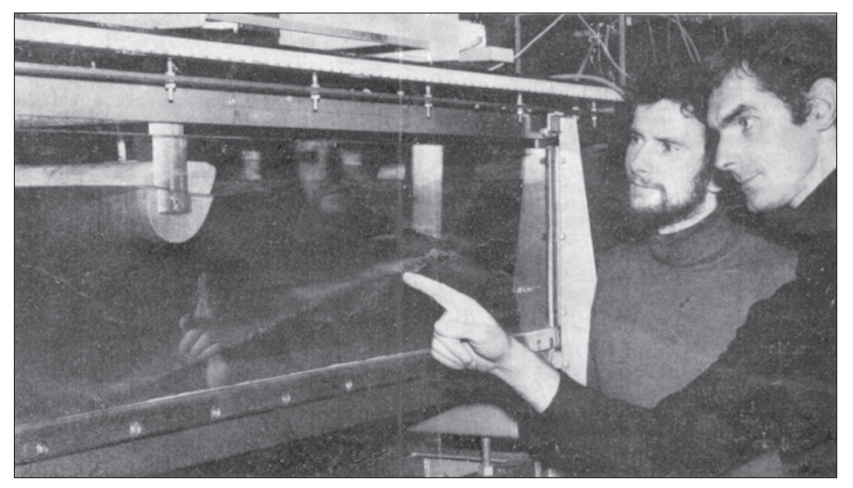

\section{Fig. 2 - Stephen Salter et David Jeffrey devant un prototype houlomoteur en 1974}

Photo Jamie Taylor, University of Edinburgh.

Ayant rapidement compris les potentialités de récupération de l'énergie des vagues après le premier choc pétrolier, le Japan Agency for MarineEarth Science and Technology (JAMSTEC) se lança dès 1971 dans l'étude et la conception d'" une centrale électrique fonctionnant à l'énergie de vagues $^{40} »$. En Europe, un programme anglais sur l'énergie des vagues est lancé en $1975^{41}$. Une première phase de 1976 à 1978 est dotée de 2,5 millions de livres ${ }^{42}$. Il s'agit pour les Britanniques « de prendre une "assurance sur l'avenir" dans l'hypothèse où pour des raisons techniques, sociologiques

40. Jean-Marie Thierry, L'énergie des vagues : la RひD japonaise, Société franco-japonaise d'océanographie, février 1990, p. 61-65.

41. P. G. Davies et al., Wave Energy: The Department of Energy's $R$ \& D Programme 1974-1983, Department of Energy Energy Technology Support Unit, 1985.

42. Gilbert Damy, Michel Gauthier, "Production d'énergie à partir de la houle », op. cit. 
ou politiques, le nucléaire ne permettrait pas au-delà de l'horizon 2000 de compenser l'épuisement prévisible des ressources en combustibles fossiles $^{43}$ ». De nombreux mécanismes sont alors proposés, testés en bassin et en mer, le plus connu étant peut-être les fameux Canards de Salter (Salter's Ducks), mis au point à l'Université d'Edimbourg (Fig. 2). On peut aussi citer le Cockerell Raft, inventé par Sir Christophe Cockerell (mieux connu pour avoir inventé l'aéroglisseur) et testé dans le Solent en 1978 ${ }^{44}$. Ces systèmes "visent à concurrencer directement les centrales terrestres du charbon à bon marché ou l'énergie nucléaire ${ }^{45}$ ». Par la suite, le département britannique de l'énergie commanda une étude de faisabilité pour le déploiement de $2000 \mathrm{MW}$ d'énergie houlomotrice ${ }^{46}$.

Cette émulation internationale, et plus particulièrement de l'autre côté de la Manche, encourage les acteurs français à s'intéresser au houlomoteur. En 1978, une étude de synthèse est effectuée par le Centre océanographique de Bretagne à la demande de la Direction générale du Centre national pour l'exploitation des océans (CNEXO). Des machines prototypes sont testées au laboratoire de Maisons-Alfort ${ }^{47}$. En janvier 1981, un concours "Houle » est lancé par l'Agence nationale de valorisation de la recherche (ANVAR) et le CNEXO durant l'émission télévisée "Thalassa ", avec pour objectif de promouvoir l'utilisation de l'énergie des vagues pour alimenter des installations réclamant une énergie moyenne (pompage, bouées, irrigation, dessalement...). Ce concours suscite l'intérêt d'un grand nombre de participants (650) plus ou moins sérieux ${ }^{48}$. Les deux lauréats gagnent la réalisation d'une maquette et d'un essai en bassin de houle dans les installations de l'ENSM. Au même moment la société UIE teste en bassin en collaboration avec le CNEXO deux versions d'un système hydropneumatique appelées WHEP1 et WHEP2. La société Bertin imagine quant à elle un dispositif immergé fonctionnant en circuit fermé. Néanmoins, alors qu'à l'étranger on semble s'aventurer hardiment dans cette voie en se lançant d'ores et déjà dans une phase expérimentale, la France reste

43. Ibid., p. 39.

44. David Ross, Power from the Waves, op. cit.

45. "Un prototype de centrales à partir des vagues ", Énergie Magazine, mars 1985, p. 57.

46. Rendel Palmer Tritton, Kennedy Donkin, United kingdom wave energy program - Consultants' 1981 Assessment, Report, Department of Energy, UK, 1982.

47. Georges Moguilny, "Emploi de la houle aléatoire dans les essais de laboratoire ", La Houille Blanche, 1986, n 4-5, p. 377-383.

48. «Les énergies des mers », Clés pour demain, TF1, 19 janvier 1981. 
plus prudente. Pour le CNEXO, « il ne s'agit pas de renouveler l'expérience de l'usine marémotrice de la Rance, exemplaire du point de vue technique, mais pas rentable ${ }^{49}$ ». L'évaluation de l'ampleur du gisement énergétique montre de plus que « la France métropolitaine présente une ressource moyenne $(10 \mathrm{~kW} / \mathrm{m})$ qui semble trois fois moins dense qu'en Écosse, Irlande ou Norvège. Le potentiel houlomoteur métropolitain est difficile à exploiter du fait du marnage important et d'une puissance très variable ${ }^{50} »$. En matière d'énergies marines, la France semble alors nourrir plus d'espoir concernant l'énergie marémotrice ${ }^{51}$ et l'énergie thermique des mers $^{52}$.

Les difficultés économiques des années 1980 et le contre-choc pétrolier mettent rapidement fin à la plupart des projets houlomoteurs :

Les Norvégiens, qui ont construit en 1984 à Bergen, une unité de $50 \mathrm{~kW}$ croient toujours en la possibilité de faire marcher de manière rentable des centrales à houle de 0,5 à $50 \mathrm{MW}$. Mais les Britanniques, qui figuraient en tête des recherches, abandonnèrent la partie en 1983 pour des raisons politiques. Les Français n’ont guère plus d'espoir, et plusieurs projets, étudiés parfois dès 1944, dorment dans les cartons ${ }^{53}$.

Au Royaume-Uni, " dans le climat économique et politique actuel, parler d'un tel projet est assez malvenu ${ }^{54}$ ». En France, les recherches sur les " énergies prototypes » sont stoppées en $1986^{55}$. La consolidation de la trajectoire

49. "Apprivoiser les vagues : du rêve à la réalité ", L'Usine Nouvelle, février 1981.

50. Philippe Marchand, "Les énergies marines renouvelables ", Paris, CNEXO, 18 décembre 1981.

51. Le 14 février 1982, le ministre délégué à l'énergie Edmond Hervé demande à EDF d'étudier la possibilité de développement une structure marémotrice d'une zone de $200 \mathrm{~km}^{2}$ entre Coutainville, les îles Chausey et Granville. «Une usine marémotrice sur la côte ouest du Cotentin ? ", Le Marin, 22 octobre 1982.

52. En France, entre 1982 et 1985, le groupement d'intérêt économique Ergocéan, unissant l'IFREMER à des industriels comme Spie-Batignolles ou Alstom, consacra 70 millions de francs à des études portant sur une centrale énergie thermique des mers (ETM) pilote à Tahiti. Pierre Blanchard, Avant-projet - Tahiti Centrale ETM. 5 MW, Retombées potentielles pour le territoire de la Polynésie française, IFREMER, juin 1986.

53. "L'indomptable énergie des flots ", L’Express, 10 juin 1988.

54. "Royaume-Uni : projets de centrales hydrauliques utilisant l'énergie des vagues ", Navires Ports et Chantiers, octobre 1986.

55. EDF arrête le financement de la centrale solaire Thémis en septembre 1986. Abandon du projet énergie thermique des mers (5 MW) en Polynésie française. Michel Gauthier, "L'énergie 
du nucléaire civil au cours des années 1980 engendre de fortes inerties limitant l'allocation de ressources vers la recherche de nouvelles technologies énergétiques. Bien qu'à la marge du système électrique et privée de subventions nationales, la communauté scientifique continua dans les années 1990 à rester active sur le sujet de l'énergie des vagues grâce à l'Union Européenne, avec le soutien notamment de la DG XII (Direction chargée de la science, de la recherche et du développement) présidée par Édith Cresson. Ainsi, quatre grandes conférences internationales ont lieu en Europe (Edimbourg 1993 ; Lisbonne 1995 ; Patras 1998 et Aalborg 2000) et des prototypes sont financés au titre des programmes communautaires : le projet danois Wave Dragon (36 kW), le projet Limpet en Écosse $(500 \mathrm{~kW})$ et le projet portugais de l'île Pico $(400 \mathrm{~kW})$ pour ne citer que les plus connus. La dynamique du houlomoteur ne s'est donc pas totalement arrêtée dans les années 1980 au Royaume-Uni et au Danemark (elle a vécu à l'état latent), ce qui va être d'une importance notable dans la résurgence du houlomoteur dans les années 2000.

\section{Le temps de l'innovation et de la crise environnementale (1998-2014)}

Le système énergique, après une phase de (re)consolidation par les énergies fossiles, entre dans une nouvelle phase de déstabilisation au début des années 2000, motivée par des enjeux énergétiques et environnementaux. Ce contexte encourage les États à réactiver des trajectoires anciennes pour produire de l'électricité et donne un nouvel élan aux projets de la filière houlomotrice. Surtout, les nouvelles technologies informatiques (logiciels de simulation) et les dernières techniques mises au point par l'industrie pétrolière offshore, les chantiers navals et la pêche, permettent aux ingénieurs d'imaginer des machines plus performantes et résistantes.

Les savoir-faire et compétences acquis par le Royaume-Uni depuis les années 1970 lui permettent de relancer rapidement des projets houlomoteurs et de se positionner comme leader dans la compétition technologique. L'objectif est alors double : ressortir des cartons les concepts hérités des années 1980 (certains industriels parlent de technologies "sur étagères »)

thermique des mers, une énergie renouvelable oubliée mais pleine d'avenir... ", La Revue Maritime, mars 2006, $n^{\circ} 475$. 
et les remettre à jour à coups d'innovations incrémentales. Il s'agit ici de mettre en place une stratégie d'optimisation du patrimoine technologique existant ${ }^{56}$.

Tout comme les compagnies pétrolières et les banquiers, le gouvernement britannique tend à nouveau une oreille attentive aux ingénieurs impatients d'obtenir rapidement des subventions. Ce comportement peut être expliqué par le fait que les firmes ont été séduites par l'important potentiel d'application et d'évolution des technologies houlomotrices, et parce qu'elles ont bénéficié des interventions publiques résultat des nouveaux objectifs environnementaux, comme l'explique un chef de projet : « Nous avons décidé de créer notre société lorsqu'en 1998, on a annoncé que toute forme d'électricité produite par les vagues en Écosse serait rachetée à prix subventionné, et ça, c'était le premier signal d'encouragement politique ${ }^{57}$. " Cette tendance est confirmée par le déploiement d'un grand nombre de démonstrateurs en mer, à des échelles variées.

Leader incontestable en récupération de l'énergie des vagues, le système écossais Pelamis, constitué de quatre cylindres flottants reliés par des articulations qui captent l'énergie de la houle, est alors le seul système arrivé au stade industriel parmi la dizaine de projets d'énergie houlomotrice développés en Europe ${ }^{58}$. En 2009, l'émission «Thalassa » consacre un reportage sur Pelamis, la " première centrale houlomotrice au monde " ${ }^{59}$. Le serpent de mer Pelamis est alors présenté comme un modèle de réussite énergétique : "C'est ainsi que ce drôle d'engin a été jugé viable économiquement et technologiquement ${ }^{60}{ }^{\prime}$ (Fig. VI, cahier couleur). En 2003, le European Marine Energy Center (EMEC) est créé avec pour objectif de faciliter le développement des énergies marines renouvelables en offrant la possibilité de tester les prototypes houlomoteurs en conditions réelles, au nord de l'Écosse. En Finlande, la société AW-Energy créée en 2002 développe la technologie Waveroller. Au Danemark, dans la suite du Bolgekraftprogram de 1998-2002, une activité importante autour de l'énergie des vagues est

56. Thomas Durand, "Management stratégique de la technologie : dix enseignements ", Revue Futuribles, novembre 1989, n 137 , p. 39-53.

57. Propos du chef de projet pour Pelamis, Thalassa, France 3, 13 novembre 2009.

58. «Un projet de centrale houlomotrice », Le Marin, 23 décembre 2005.

59. Émission Thalassa, France 3, 13 novembre 2009.

60. Expérimentation d'un générateur d'électricité basé sur les courants marins, journal télévisé de 20 h, France 2, 14 janvier 2008. 
relancée. En Espagne, en 2007 et 2008, seize turbines furent intégrées à la nouvelle digue du port de Mutriku. La centrale fut inaugurée en 2011 $(259 \mathrm{~kW})$. La première centrale à vagues de monde en vue de la production d'énergie est inaugurée le 28 septembre 2007 sur la plage de l'Aguçadoura au Portugal ${ }^{61}$. Notons ici que la dynamique ne se limite pas à l'espace européen (Ocean Power Technologies aux États-Unis et la société Carnegie Wave Power ${ }^{62}$ en Australie par exemple). En 2008, on compte quarante-six projets de capteurs de l'énergie des vagues dans le monde ${ }^{63}$.

Cette émulation internationale ne laissa pas indifférent les acteurs français : « Nous avons commencé à regarder le sujet houlomoteur à partir de 20052006. Nous sentions à l'époque une dynamique générale de marché, en particulier au Royaume-Uni, avec l'ouverture d'un certain nombre de programmes de financement et de support. Cette émulation générale nous a interpellés ${ }^{64}$. " Aussi, le début des années 2000 et 2010 a été marqué par un fort investissement des centres de recherche, des industriels et des énergéticiens pour le développement de l'énergie houlomotrice. Présenté comme " la centrale électrique marine du futur ${ }^{65}$ " par les médias, le projet SEAREV, issu de l'aboutissement de vingt ans de recherche des laboratoires de Centrale Nantes, est lancé en 2002. EDF EN et DCNS développent un premier projet basé sur la technologie CETO (Australie) à la Réunion. En 2011, Alstom rachète 40 \% de la société écossaise AWS Ocean Energy. Ces différents projets doivent être replacés dans un contexte plus global qui est celui de la généralisation d'une politique publique basée sur les démonstrateurs au tournant des années $2000^{66}$. Cette dynamique scientifique et industrielle s'accompagne d'une dynamique territoriale. Les objectifs du Grenelle de l'environnement encouragent en effet les territoires à se lancer dans des projets technologiques innovants dans le domaine de l'énergie.

61. La ferme comporte trois convertisseurs Pelamis convertissant l'énergie des vagues (mouvement des ondes de surface) de l'océan en électricité, pour un total de 2,25 MW puissance totale installée : http://wavepower.ek.la/pelamis-p488823, consulté le 7 novembre 2018.

62. Aujourd'hui Carnegie Clean Energy.

63. Propos de Patrick Chedmail, directeur de l'École centrale de Nantes, dans "L'Énergie marine testée grandeur nature ", Les Échos, 8 octobre 2008.

64. Entretien SBM Offshore, 30 mai 2017.

65. Dans 7 h 30 le Journal, France 2, 15 juin 2009.

66. François MoIsan, "Du fonds démonstrateur aux investissements d'avenir : promouvoir une offre française dans le domaine des technologies vertes ", Annales des Mines, Responsabilité et environnement, 2011, n 61 , p. 109-115. 
Un site d'essais baptisé SEMREV destiné à tester des machines houlomotrices est lancé en 2010 au large du Croisic. Dans les DOM-TOM, le potentiel des énergies marines renouvelables est alors jugé particulièrement intéressant ${ }^{67}$.

Néanmoins, cette dynamique optimiste du milieu des années 2000 va rapidement laisser la place au pragmatisme et à l'attentisme. Le houlomoteur connaît une crise de confiance à partir de 2012. Vitrine de la filière houlomotrice des années 2000, Pelamis met la clé sous la porte faute de financements et de repreneur, représentant un "véritable traumatisme pour la communauté énergie des vagues ${ }^{68} »$. Le second choc fut la faillite du challenger Oyster, seulement un an plus tard. Les déboires de ces PME pionnières constituent un sérieux revers pour le secteur du houlomoteur car elles étaient parvenues il y a quelques années à séduire de grands industriels et à s'assurer leur soutien financier et technologique ${ }^{69}$. En France, le nombre des acteurs français prêts à développer cette filière s'est fortement réduit ces dernières années après les défections de trois " champions nationaux ». Naval Group a décidé de mettre le houlomoteur en veille pour se concentrer sur l'hydrolien, l'ETM et l'éolien flottant. Le projet Houles Australes, développé avec EDF EN au large de la Réunion est arrêté fin 2013 lorsque le prototype CETO $(150 \mathrm{~kW})$ a été emporté par le cyclone Bejisa. Enfin Alstom a interrompu en 2014 ses investissements dans le houlomoteur et a revendu $40 \%$ des parts qu'il détenait depuis 2011 dans la britannique AWS Ocean EnergyRenewables. Sont également interrompus les projets Bilboquet, de la société française d'architecture navale et d'ingénierie marine D2M, et SEAREV, de l'École centrale de Nantes. Notons enfin que cette crise de confiance ne se limite pas à la France ${ }^{70}$.

67. Sylvain Roche, «L'énergie thermique des mers dans les Outre-mer français : un enjeu stratégique de territoire ?", Études caribéennes, hors-série 1, juillet 2018, en ligne : http://journals. openedition.org/etudescaribeennes/11971, consulté le 16 octobre 2018.

68. Aurélien Babarit, Ocean Wave Energy Conversion, op. cit., p. 95.

69. "Les énergies marines au creux de la vague ", Libération, 30 janvier 2015.

70. «Scottish government accused of abandoning wave power », The Guardian, 9 décembre 2014. 


\section{Le temps de la nécessaire rupture systémique (depuis 2014)}

Après un réveil soudain au début des années 2000, l'énergie des vagues se situe aujourd'hui dans une phase délicate. Après des années de simulations sur ordinateur puis d'essais sur des maquettes en laboratoire, les prototypes grandeur nature les plus avancés des années 2000 n’ont pas dépassé la phase des essais menés dans les conditions de mer des Orcades. Le processus de " marinisation " technologique s'est notamment heurté au problème de la résistance mécanique des systèmes houlomoteurs qui doivent supporter des tempêtes imprévisibles et des variations colossales de puissance. Il existe en effet un paradoxe entre la simplicité de la représentation mentale de l'énergie des vagues et la complexité de son exploitation technologique.

L'énorme quantité de structure passive des machines (le blindage) qui n'a pas d'autre intérêt que de résister aux vagues extrêmes et de protéger la structure active (qui permet la récupération d'énergie) est certainement l'une des contraintes technologiques les plus importantes. La taille implique en effet des efforts proportionnels au volume de la machine. Ce problème technique lié à la taille était déjà mentionné comme un verrou contraignant dès les années 1970 : "Pour que les investissements dans l'énergie des vagues deviennent rentables, on est condamné au gigantisme. Pour obtenir un gigawatt, il faudra équiper environ 100 kilomètres de côtes ${ }^{71}$ ". En 2006, le Pelamis est qualifié de «monstre flottant de 700 tonnes $^{72}$ ». Le SEAREV est quant à lui une machine pesant près de 1000 tonnes.

Cette complexité du domaine technologique s'est directement répercutée sur le coût économique. Les partisans de l'énergie houlomotrice ont eu cette difficile mission de prouver que leurs dispositifs sont capables de résister aux tempêtes les plus violentes et également de produire de l'énergie à un prix qui soit compétitif avec celui obtenu par les centrales fonctionnant au charbon, au pétrole ou à la fission nucléaire :

Sur le plan technique, le problème essentiel est de trouver une méthode rentable pour la conversion d'une fraction de l'énergie

71. "Les énergies de la mer ", Le Monde, 13 juillet 1980.

72. «L'énergie des vagues fait surface », L'Usine Nouvelle, 20 avril 2006. 
contenue dans le mouvement des vagues en énergie mécanique, hydraulique ou pneumatique qui, à son tour, peut être utilisée pour produire de l'électricité à une échelle substantielle dans les conditions inhospitalières du grand large ${ }^{73}$.

L'énergie houlomotrice a aussi été mise en concurrence avec d'autres énergies renouvelables plus matures et mieux maitrisées bénéficiant d'effets de taille et de rendements croissants d'adoption, et d'une base de connaissances mieux constituée. La comparaison des différents $\mathrm{kWh}$ électriques (benchmarking) va ici clairement mettre en avant le surcoût économique du houlomoteur par rapport aux technologies énergétiques concurrentes (market barrier) :

L'utilisation de l'énergie de la houle le long des côtes conduirait à des coûts dix fois supérieurs à celui d'un barrage hydraulique ${ }^{74}$ [...] Les premières analyses économiques donnent un prix de l'énergie plus élevé que pour l'énergie nucléaire, le rapport des coûts étant compris entre 1 et $3^{75}[\ldots]$ Des modèles (prototypes houlomoteurs) au 1/10 auraient montré qu'ils pouvaient produire de l'électricité à 2 à $3 \mathrm{~F} / \mathrm{kWh}$. C'est encore cher comparé à l'électricité générée par des centrales au charbon $(0,35 \mathrm{~F} / \mathrm{kWh})$ ou nucléaires $(0,25 \mathrm{~F} / \mathrm{kWh})^{76}[\ldots]$ L'exploitation de l'énergie des vagues doit être également compétitive avec les autres formes d'énergies renouvelables telles que l'énergie éolienne, la géothermie et la biomasse qui se révèlent de plus en plus prometteuses ${ }^{77}$.

De plus, la grande diversité technologique depuis les années 1970 a empêché l'affirmation progressive d'une technique dominante (dominant design), reconnue par le marché comme le design du produit ou du service qui répond au mieux aux besoins des consommateurs. La filière est toujours actuellement dans une phase de prototypage-démonstration et n’a

73. Conférence mondiale de l'énergie, Perspectives énergétiques mondiales à l'horizon 2020 : rapport, Paris, Éditions techniques et économiques, 1979, p. 134.

74. Communication de Marcel Barrère, directeur scientifique à l'Office national d'études et de recherches aéronautiques, ONERA au colloque «A.X Techniques de Pointe : Quelle place pour la France ? ", 17-18 mars 1982, Société amicale des anciens élèves de l'École polytechnique, Paris. 75. André Brin, Océan et énergie, Paris, Technip, 1979, p. 106.

76. "Apprivoiser les vagues : du rêve à la réalité ", L'Usine Nouvelle, février 1981.

77. «Énergies des vagues : les Britanniques y croient ", Innovation et Produits Nouveaux, Presses de l'Innovation, février 1981, p. 21. 
pas encore convergé sur un dominant design ${ }^{78}$. Ce maintien de la diversité retarde la sélection d'un standard (processus de standardisation) et la mise en place des rétroactions positives qui lui sont associées. Cette diversité technologique a de plus comme effet pervers d'avoir éclaté la recherche et développement. De nombreuses petites équipes de recherche et développement se sont développées en parallèle, et très vite, les chercheurs se sont sentis en concurrence, notamment dans le cadre de l'obtention de ressources de financements de projets. Trop de concepts se sont partagé les financements publics, alors que l'industrie éolienne a par exemple depuis longtemps concentré son choix sur les turbines à axe horizontal et à trois pales $^{79}$. Ce processus de standardisation technologique s'est réalisé à la fin des années 1990 pour le solaire photovoltaïque ${ }^{80}$.

À la différence par exemple de l'hydrolien qui est présenté comme "un moulin sous-marin ${ }^{81}$ " ou une " éolienne sous-marine $e^{82}$ " issue des technologies de turbines (technologie générique), l'énergie houlomotrice n’a pas cette possibilité de créer des analogies avec des technologies antérieures ou plus matures («Les grands industriels se sentent plus à l'aise avec l'hydrolien, ça ressemble à des éoliennes, c'est plus familier [...] Au contraire, personne n'a l'intuition de ce qu'est un appareil houlomoteur, les concepts sont moins uniformes $\left.{ }^{83} »\right)$. Or, comme nous le rappelle Jacques Perrin, la capacité à susciter et à organiser des analogies joue un rôle central dans la démarche d'innovation et de standardisation (généalogie historique) ${ }^{84}$. Elles apportent un aspect rassurant, qui est celui d'une technologie mature

78. Selon le site internet de l'European Marine Energy Centre (EMEC), plus de deux cents systèmes de récupération de l'énergie des vagues étaient en cours de développement dans le monde en 2018, regroupés en huit grandes familles : http://www.emec.org.uk/marine-energy/wave-devices/, consulté le 17 octobre 2018.

79. Johannes Juul construit en 1956 la première éolienne à produire du courant alternatif. Composée de 3 pâles et d'une puissance de $200 \mathrm{~kW}$, c'est elle qui a inspiré les éoliennes d'aujourd'hui : http:// ele.aut.ac.ir/ -wind/en/pictures/juul.htm, consulté le 7 novembre 2018.

80. Philippe Menanteau, «L'électricité photovoltaïque dans l'attente de l'émergence d'un nouveau standard technologique ", Revue de l'Énergie, 1999, n 50, p. 714-718.

81. "Des moulins sous-marins ", Le Télégramme, 24 mars 1999.

82. "La plus puissante "éolienne sous-marine" est britannique ", Le Figaro, 12 avril 2008.

83. Richard YEMM, directeur général de Pelamis, dans "L'Écosse, poisson pilote des énergies marines ", Libération, 21 octobre 2013.

84. Jacques Perrin, Concevoir l'innovation industrielle, Méthodologie de conception de l'innovation, Paris, CNRS Éditions, 2001. 
et maitrisée, servant implicitement ou explicitement de référence aux décideurs et financeurs.

Aujourd'hui, le houlomoteur n'est pas sorti de l'échelle des TRL (Technology Readiness Level ou Échelle de Maturité Technologique) et donc à ce titre, il n'est pas sorti de la zone de risque ${ }^{85}$. La filière perdure dans la "Vallée de la mort " (Death Valley), cette phase critique en finance de l'innovation située entre les financements publics (faible TRL) et privés (fort TRL) ${ }^{86}$. Cette situation "d'incertitude prospective " encourage les firmes à rester dans une situation de blocage (lock-in) marquée par un phénomène d'attentisme de l'investissement pour éviter de s'engager trop hâtivement dans une direction qui se révélera économiquement sous-optimale ${ }^{87}$. L'énergie des vagues est de plus aujourd'hui directement impactée par les projections optimistes des années 2000, que ce soit en termes de calendrier, de production d'énergie ou de prix du kWh houle ${ }^{88}$. Pour bon nombre de projets, le développement se révéla plus difficile, plus coûteux, et certainement trop optimiste :

Il y a trois ou quatre ans, Aquamarine et Pelamis, les deux piliers écossais du secteur, et leurs partenaires respectifs, les électriciens écossais SSE et l'allemand E.ON, évoquaient un potentiel d'installation de 1000 mégawatts (MW) et plus en dix ans, soit la puissance moyenne d'un réacteur nucléaire français. Aujourd'hui, les deux entreprises testent encore des prototypes d'une puissante inférieure au $\mathrm{MW}^{89}$.

À ce titre, plusieurs auteurs ont en effet montré qu'un tel type de récit, dans le cas des sciences et des technologies émergentes comme les nanotechnologies ou la biologie de synthèse, est amené à jouer un rôle si central

85. Introduits par Stan Sadin de la NASA en 1974, les TRL constituent une échelle d'évaluation de la maturité d'une technologie allant de 1 à 9 . Pour franchir un nouveau TRL, des critères techniques doivent être respectés, et ce pour chaque niveau.

86. Thomas Randolph Beard, George S. Ford, Thomas M. Koutsky, Lawrence J. SpiwaK, "A Valley of Death in the innovation sequence: an economic investigation", Research Evaluation, vol. 18, 2009, Issue 5, 1, p. 343-356.

87. Yves Chalas, Claude Gilbert, Dominique Vinck (dir.), Comment les acteurs sararrangent avec l'incertitude, Paris, Édition des archives contemporaines, 2009.

88. Voir par exemple Michel Paillard, Denis Lacroix, Véronique Lamblin (dir.), Énergies renouvelables marines. Étude prospective à l'horizon 2030, Versailles, Quae, 2008.

89. «L'Écosse, poisson pilote des énergies marines », op. cit. 
qu'il n'est pas exagéré de parler de la constitution d'une « économie de la promesse $^{90} »$.

Comme nous l'avons montré auparavant, l'énergie houlomotrice a progressé ces dernières années à coups d'innovations incrémentales le long d'une trajectoire héritée des années 1970-1980. Éperonnés par ce système de contraintes à surmonter, les ingénieurs orientent désormais leurs travaux vers des systèmes cherchant à produire de l'énergie à partir de la houle avec les meilleurs rendements et prix de revient possibles, en s'appuyant sur de nouvelles connaissances extensives. Cette transition délicate vers un nouveau houlomoteur passe par une phase de divergence et de génération d'idées. De nouvelles technologies s'inspirant du vivant doivent permettre non seulement de résister plus longtemps aux conditions hostiles du milieu marin, mais aussi de mieux s'intégrer dans les écosystèmes environnants ${ }^{91}$.

La rupture systémique se trouve dans la manière d'appréhender la vague. Si les machines conventionnelles s'opposent à la vague en cherchant à l'arrêter pour capter son énergie (dans un schéma classique hérité de la pensée cartésienne de l'homme contre la nature), le nouveau houlomoteur cherche à composer avec la vague, à l'accompagner, en s'inspirant de systèmes naturels (Fig. VII, cahier couleur). La recherche de rupture autour des écomatériaux souples doit permettre de sortir du cercle vicieux $\mathrm{du}$ " gigantisme technologique " hérité du paradigme du machinisme. Le redimensionnement d'un houlomoteur du big is beautiful vers un small is beautiful marque là aussi une rupture méthodologique certaine.

Le nouveau houlomoteur est aussi " une technique de bissociations ${ }^{92}$ " qui consiste à mettre en relations des univers de référence hétérogènes pour essayer de faire jaillir des surprises créatives (interrelations technologiques). À partir de plusieurs exemples tirés de l'histoire des semi-conducteurs, de l'avion ou de lampe électrique, William Abernathy et James Utterback ont montré qu'une innovation " radicale " n’est pas réalisée ex nibilo, mais qu'elle est généralement le résultat de l'accumulation de plusieurs

90. Marc Audetat (dir.), Sciences et technologies émergentes : pourquoi tant de promesses, Paris, Hermann, 2015.

91. Le groupe Renault explore aujourd'hui la possibilité de s'inspirer du métabolisme humain pour repenser la motorisation du véhicule décarbonée de demain. Voir l'interview de Jérôme Perrin, Directeur Scientifique RENAULT à UP Magazine, juillet 2016. Vidéo en ligne : https://www. youtube.com/watch ?v =TOMcanBRvpE, consultée le 17 octobre 2018.

92. Guy Aznard, «Préciser le sens du mot "créativité" ", Synergies Europe, 2009, n 4, p. 23-37. 
innovations introduites dans le produit de manière indépendante ${ }^{93}$. Il s'agit donc bien d'encourager l'innovation par la convergence d'innovations et les synergies de domaines. Le nouveau houlomoteur ne doit pas se limiter à la production d'électricité, mais doit trouver une place dans un tissu complexe d'autres usages (extraction de minerais, dessalement de l'eau de mer, propulsion et stabilisation des navires ou plateformes, alimentation de drone marin...).

Ces nouvelles perspectives stimulantes ne doivent pas néanmoins nous faire oublier que le houlomoteur reste dans une période de latence, c'est-àdire « la durée nécessaire pour parvenir à imaginer des usages, que l'innovation technologique commence à se transformer en innovation technique, organisationnelle et sociale ${ }^{94}$ ». Michel Godet $e t$ al. ajoutent que «le temps de latence est lié aux processus de sélection des technologies les plus efficaces et à leurs améliorations, à leur simplification, à leur optimisation, à leur maîtrise, à la réduction de leur coût, et à leur combinaison en système complexe. Cela peut prendre beaucoup de temps comme dans le cas de la machine à vapeur ${ }^{95}$ ". Cet " agenda du temps long " souligne l'importance de poursuivre les efforts de recherche et développement en matière d'expérimentation et de prototypage, car eux seuls peuvent permettre de produire les connaissances à partir desquelles on peut faire reculer les limites du possible en réinterrogeant les frontières (learning by doing). Et à ce titre, la mise en œuvre de démonstrateurs de recherche reste une étape essentielle de la validation des différentes ruptures technologiques et méthodologiques $^{96}$, lorsque que les études préalables ont validé une estimation fiable du coût de l'énergie (et ainsi limiter la construction de prototypes inutiles).

Cette inscription dans le temps long pourrait être rendue possible par la création de niches, où se consolideront à l'abri du système dominant, les innovations alternatives et les réseaux d'acteurs qui leur sont liés. À ce titre Jean Bonal et Pierre Rossetti nous rappellent que la technologie du

93. William Abernathy, James Utтerback, "Patterns of industrial innovation”, Technology Review, 1978, 80, 7, p. 2-9.

94. Norbert Alter, "L'innovation : un processus collectif ambigu ", in Norbert Alter (dir.), Les logiques de l'innovation. Approche pluridisciplinaire, Paris, La Découverte, 2002, p. 21.

95. Michel Goet, Philippe Durance, Marc Mousli, Créativité et innovation dans les territoires, rapport du Conseil d'analyse économique, Paris, La Documentation française, 2010, p. 118.

96. François Moisan, "Du fonds démonstrateur aux investissements d'avenir : promouvoir une offre française dans le domaine des technologies vertes ", Annales des Mines, Responsabilité et environnement, 2011, n 61, p. 109-115. 
photovoltaïque s'est d'abord développée, dès 1975, par la conquête progressive de niches de marchés, à travers des applications professionnelles (bornes téléphoniques d'appels d'urgence, télédétection, alarmes, balisages maritimes, relais de télécommunication, etc. $)^{97}$. Pour le houlomoteur, on peut notamment penser aux territoires insulaires situés en dehors des grands réseaux d'énergie continentaux qui privilégient les productions centralisées d'énergie. Pour autant, il semble aujourd'hui vraisemblable de considérer qu'à l'heure actuelle, le coût des premiers projets houlomoteurs raccordés sur des réseaux iliens ne sera pas inférieur à $600 € / \mathrm{MWh}$. À titre de comparaison, il se trouve que c'est à peu près le prix du kWh électrique dans les îles les plus isolées du Pacifique (Kiribati, Vanuatu et Cook's).

\section{Conclusion}

L'énergie houlomotrice est loin d'être une énergie nouvelle, comme elle est si souvent présentée. C'est tout son paradoxe : c'est l'une des filières EMR aujourd'hui les moins matures alors que c'est celle où la créativité est la plus ancienne. L'idée d'exploiter l'énergie des vagues s'est toujours heurtée à de gros problèmes technico-économiques. La trajectoire du houlomoteur nous montre que ce qui est possible sur le plan technologique ne l'est pas forcément sur un plan économique. Si l'énergie des vagues entre aujourd'hui dans une logique environnementale, il lui reste ce défi de s'intégrer dans la logique socio-économique du marché hautement concurrentiel de l'énergie. En citant Franco Romerio : «L'utopie est utile si elle fournit l'énergie nécessaire pour faire décoller des projets et assurer des percées technologiques. Toutefois, la réalité ne doit jamais être perdue de vue et la rigueur des faits ne doit être en aucun cas être abandonnée ${ }^{98}$ ". De véritables ruptures technologiques semblent donc à ce jour indispensables pour déverrouiller la filière, convaincre les indécis et ainsi impulser une nouvelle dynamique vertueuse. L'énergie des vagues doit atteindre la phase ultime, à savoir le stade paradigmatique, qui se caractérise par la standardisation de la technologie ${ }^{99}$. Pour y arriver, il doit se construire

97. Jean Bonal, Pierre Rossetti, Énergies alternatives, Paris, Omniscience, 2007.

98. Franco Romerio, Les controverses de l'énergie, Lausanne, Presses Polytechniques Romandes, 2008, p. 106.

99. David Teece distingue la phase pré-paradigmatique durant laquelle la concurrence au sein d'une industrie s'exprimerait essentiellement entre diverses options technologiques, de la phase paradigmatique proprement dite, au cours de laquelle, un design dominant ayant émergé, la compétition 
un environnement technico-économique favorable lui permettant de « maturer » dans de bonnes conditions (le marché des territoires insulaires par exemple).

La trajectoire de l'énergie des vagues nous confirme que le processus d'innovation n'a rien de linéaire, et ceci pour au moins deux raisons. Tout d'abord elle s'inscrit dans un cycle d'apparition-disparition-réapparition tel que l'avait observé David Edgerton ${ }^{100}$. Les évolutions économiques et les nouveaux impératifs environnementaux ont requalifié le houlomoteur au tournant des années 2000 avant que la réalité technico-économique ne le rattrape. La progression des performances est encore insuffisante pour assurer sa compétitivité avec les technologies de production d'électricité plus matures qui connaissent un rythme de progression plus important (comme l'énergie solaire par exemple). Aussi, la trajectoire s'inscrit dans un cycle de type hype-hope-disappointment tel qu'identifié par Gideon Gartner $^{101}$ ou Victor Scardigli dans Les Sens de la Technique ${ }^{102}$. Dans la vision du monde selon Gartner, la visibilité des nouvelles technologies grimpe rapidement vers des sommets sous l'effet d'une excitation initiale pour la nouveauté. Cette phase est suivie par un "creux de la désillusion ", phase au cours de laquelle les attentes démesurées sont confrontées à la réalité. Le modèle suppose donc qu'il existe un décalage entre la dynamique des opinions sociales, marquées par la vitesse de construction des promesses technologiques, et les processus d'innovation dans le domaine énergétique marqués par de fortes inerties.

Aussi plus généralement, la situation actuelle du houlomoteur poserait la question de la corrélation du temps du projet technologique et celui de l'utopie, de la corrélation du temps de la science et de celui de l'industrie. Comment concilier des objectifs de rentabilité économique à court et moyen terme au sein d'un secteur industriel (l'énergie) qui est par nature

\footnotetext{
s'exprimerait en termes d'améliorations incrémentales; tandis que la concurrence par les prix reprendrait ses droits. David Teece, "Profiting from technological innovation: implications for integration”, Research Policy, 1986, 15, p. 285-305.

100. David Edgerton, Quoi de neuf? Du rôle des techniques dans l'histoire globale, Paris, Seuil, 2013.

101. Alex Linden, Jackie Fen, Understanding Gartner's Hype Cycles, Strategic Analysis Report, 30 mai 2003.

102. Victor Scardigli, Les Sens de la Technique, Paris, PUF, 1992.
} 
celui du temps long ${ }^{103}$ ? Sur ce point Patrice Flichy souligne « qu'au démarrage du processus, on peut placer la fonction subversive de l'utopie qui permet d'explorer la gamme des possibles. C'est sans doute l'une des phases les plus inventives, mais aussi la plus brouillonne. Les innovateurs imaginent un grand nombre de dispositifs techniques qu'ils proposent d'utiliser dans les domaines les plus variés de l'activité sociale ${ }^{104}$ ». En 1976, dans son ouvrage devenu célèbre The Poverty of Power : Energy and the Economic Crisis, Barry Commoner nous décrivait l'utopie solaire comme la solution énergétique du XXI ${ }^{\mathrm{e}}$ siècle. Il est aujourd'hui frappant de voir que cette utopie solaire est devenue une réalité bien concrète ${ }^{105}$.

\section{Les auteurs}

Sylvain Roche est doctorant CIFRE en économie de l'innovation à la Région Nouvelle-Aquitaine, Groupe de recherche en économie théorique et appliquée (GREThA), université de Bordeaux. Contact : sylvain.roche@u-bordeaux.fr

Alain H. Clément est ingénieur chercheur en hydrodynamique, équipe Énergies Marines et Océan, Laboratoire de recherche en hydrodynamique, énergétique et environnement atmosphérique (LHEEA).Contact : Alain.Clement@ec-nantes.fr

Aurélien Babarit est ingénieur chercheur en hydrodynamique, équipe Énergies Marines et Océan, Laboratoire de recherche en hydrodynamique, énergétique et environnement atmosphérique (LHEEA). Contact : aurelien.babarit@ec-nantes.fr

Christophe Bouneau est professeur d'histoire contemporaine, Centre d'études des mondes moderne et contemporain - EA 2958, université Michel de Montaigne. Contact : christophe.bouneau@wanadoo.fr

\footnotetext{
103. En matière d'énergie, les temps d'incubation s'expriment en décennies, voire en siècles : par exemple environ 120 ans séparent les travaux de Franklin sur la foudre et la mise au point de la dynamo par Gramme, tandis qu'on en compte près de 60 entre la découverte de la radioactivité et le premier couplage.

104. Patrice Flichy, "La place de l'imaginaire dans l'action technique. Le cas de l'internet ", Réseaux, 2001/5, nº 109, p. 67.

105. Barry Commoner, The Poverty of Power Energy and the Economic Crisis, New York, Random House Inc, 1976.
} 